\title{
REVIEW
}

\section{Prostate cancer research in China}

\author{
Shan-Cheng Ren*, Rui Chen* and Ying-Hao Sun \\ Prostate cancer (PCa) research in China has been on a rocketing trend in recent years. The first genome-wide association study (GWAS) \\ in China identified two new PCa risk associated single nucleotide polymorphisms (SNPs). Next generation sequencing is beginning to \\ be used, yielding novel findings: gene fusions, long non-coding RNAs and other variations. Mechanisms of PCa progression have been \\ illustrated while various diagnosis biomarkers have been investigated extensively. Personalized therapy based on genetic factors, \\ nano-medicine and traditional Chinese medicine has been the focus of experimental therapeutic research for PCa. This review intends \\ to shed light upon the recent progress in PCa research in China and points out the possible breakthroughs in the future.
} Asian Journal of Andrology (2013) 15, 350-353; doi:10.1038/aja.2013.37; published online 22 April 2013

Keywords: prostate cancer (PCa); genome-wide association study (GWAS); single nucleotide polymorphisms (SNPs); China

\section{INTRODUCTION}

Prostate cancer $(\mathrm{PCa})$ is currently the second most frequently diagnosed cancer worldwide, causing 258000 deaths annually. ${ }^{1}$ Although the incidence of PCa in China was reported to be 1.6/100 000 per year, ranking one hundred seventieth among all nations, ${ }^{2}$ its incidence in China has recently increased substantially, ${ }^{3}$ with an estimated incidence of $10 / 100000$ in 2010 compared with $1.71 / 100000$ in $1993 .^{4}$ With the implementation of more comprehensive screening programmes in China, the incidence rate of PCa may rapidly increase in the future.

The number of PCa research projects by Chinese investigators has increased substantially in recent years. Led by those institutes with notable achievements, the quality of published articles by Chinese investigators is also increasing. The number of published studies doubled from 2008 to 2012; however, the number of citations to these studies increased considerably from about 300 to about 8000 (statistics from the Web of Science). Novel technologies, such as genome-wide association study (GWAS) and next-generation sequencing, are beginning to be used in China, yielding notable achievements in elucidating the aetiology, mechanisms of progression, diagnostic biomarkers and therapeutic targets of PCa. This review highlights recent progress and discusses possible future breakthroughs in PCa research in China.

\section{MAJOR ACHIEVEMENTS}

\section{Chinese GWAS in PCa}

Recently, through the joint efforts of a number of researchers, we reported the first GWAS in Chinese PCa patients. ${ }^{5}$ This is the first confirmation of the unique characteristics of Chinese PCa in a large population. A total of $4484 \mathrm{PCa}$ patients and 8934 healthy controls were involved in the study, and two new loci, 9q31.2 and 19q13.4, were found to be significantly associated with PCa risk. The rs103294 marker at $19 q 13.4$ is in strong linkage equilibrium with a $6.7-\mathrm{kb}$ germline deletion that removes the first six of seven exons in LILRA3, a gene regulating the inflammatory response, and is significantly associated with the mRNA expression of LILRA3 in T cells. Our study provides the first evidence of a genetic difference between Chinese and Caucasian PCas.

Several Chinese researchers have investigated the underlying mechanisms by which single-nucleotide polymorphisms (SNPs) are involved in prostate tumourigenesis. Lu et al. ${ }^{6}$ utilized ChIP-on-chip analyses to demonstrate that regions containing androgen receptor (AR)binding sites are significantly enriched for PCa risk-associated SNPs. It has also reported that one-third of the reported 33 genes in the Caucasian GWAS are AR-related. The findings suggest that some PCa-associated SNPs act through an androgen-mediated mechanism to influence PCa risk. ${ }^{6,7}$

Although confirmation and functional research based on Chinese GWAS are ongoing, GWAS and their potential applications have not yet been fully explored. Discovering the biological and clinical significance of GWAS results has been a new arena for those enthusiastic scientists. Studies have shown that SNPs can help to increase the positive prediction values for patients who intend to undergo prostate biopsies, significantly reducing the number of unnecessary biopsies. ${ }^{8}$ Furthermore, SNPs showed great value in the field of pharmacogenomics and personalized medicine. For advanced renal-cell carcinoma patients undergoing first-line sunitinib therapy, similar personalized therapies will be offered soon. Researchers have found two SNPs that are correlated with decreased sunitinib response and tolerability. ${ }^{9}$ For patients with these genetic polymorphisms, it would be advantageous to take decreased doses or to forgo this therapy completely. Further studies on PCa and the sunitinib research should be carried out to offer personalized treatments.

\section{Gene fusion}

The gene fusions between transmembrane protease serine 2 (TMPRSS2) and E26 (ETS) transcription factors are cancer-specific biomarkers in $\mathrm{PCa}$, and such fusions are the most common genetic events in $\mathrm{PCa}$, accounting for $90 \%$ of PCa fusions. ${ }^{10}$ Since the first report of PCa gene 
fusion in 2005, its role in diagnosis has been extensively studied. ${ }^{11}$ According to a multicentre study in North America, the prevalence of TMPRSS2-ERG gene fusion is $46 \%$ in patients undergoing biopsies. ${ }^{12} \mathrm{In}$ Chinese PCa patients, we carried out an RNA sequencing test that revealed variation in the transcriptome and indicated a much lower prevalence of TMPRSS2-ERG. Only $21.4 \%$ and $18.5 \%$ of patients in the discovery and validation periods harboured this variation. ${ }^{13}$ Such findings are consistent with previous reports on PCa patients in China, ${ }^{14}$ as well as those in Japanese and Korean populations. ${ }^{15-17}$ Two novel gene fusions, CTAGE5-KHDRBS3 and USP9Y-TTTY15, occurred frequently in our patient cohort; ${ }^{13}$ further validation in a larger and multicentre population is warranted. These differences may be further utilized in exploring the unique Chinese mechanism upon tumour onset and progression, as a study has confirmed a possible alternative path of carcinogenesis in gene fusion and PTEN inactivation. ${ }^{18}$

The occurrence of rearrangements has been explained by the following genomic, cellular and environmental changes: spatial proximity, cellular stress, inappropriate repair or recombination, and DNA sequence and chromatin features. ${ }^{19}$ The differences between Chinese and Western patients may be explained by the difference in one or more of these changes. Potential environmental differences include variation in red meat intake and in genetic backgrounds, which influence AR sensitivity. Interaction between genetic and environmental factors most likely contributes to the differences over a long and complex transition.

\section{Diagnostic biomarkers and improved imaging technologies}

Although prostate-specific antigen (PSA) has been the epoch-making marker for PCa screening and diagnosis, it is not perfect. Advances in DNA and RNA sequencing have enabled diagnostic markers to enter the era of '-omics'. ${ }^{20}$ SNPs, PCA3, microRNAs, PSA isoforms, long non-coding RNAs and TMPRSS2-ERG gene fusion are under extensive investigation.

Chinese researchers used a panel of 33 SNPs to calculate a genetic score as a diagnostic factor in a Chinese population, which showed a significant difference between the results of patients with PCa and healthy controls. ${ }^{21}$ The area under the curve for the genetic score was 0.604 for the risk prediction of PCa. Another study further combined the protein-based and metabolite-based factors in urine as a useful model for diagnosis. The expression patterns of PCA3, TMPRSS2-ERG, Annexin A3, Sarcosine and a panel including these biomarkers were significant predictors of PCa both in patients with PSA 4-10 $\mathrm{ng} \mathrm{ml}^{-1}$ and in all patients. ${ }^{22}$ Using an Illumina miRNA microarray, Chinese researchers identified five possible microRNAs with the ability to differentiate among healthy, benign and cancerous prostates. ${ }^{23}$ Our group found that intron 4 retention in PSA and exon skipping in AMACR were highly specific for PCa. Both the alternatively spliced transcripts and the proteins may serve as new potential diagnostic biomarkers for $\mathrm{PCa}{ }^{24}$

New imaging technology has improved PCa detection. It has been reported that contrast-enhanced ultrasonographic microflow imaging could significantly improve the detection accuracy rate of PCa in men with PSA values $4-10 \mathrm{ng} \mathrm{ml}^{-1}$ greater than conventional ultrasound. ${ }^{25}$ The sensitivity, specificity and accuracy were $70.3 \%, 82.5 \%$ and $80.3 \%$, respectively. Another study challenged conventional computed tomography and magnetic resonance imaging using susceptibility-weighted imaging. ${ }^{26}$ Susceptibility-weighted imaging was demonstrated to detect a greater number of prostate haemorrhages than computed tomography and magnetic resonance imaging and was better able to distinguish PCa from benign prostatic hyperplasia. Novel imaging technologies can and likely will soon offer more accurate diagnoses as a result of rapid technological innovation, such as molecular imaging.

\section{Mechanism of PCa progression}

The progression of $\mathrm{PCa}$ is mainly related to two events: metastasis and the development of castration-resistant prostate cancer (CRPC). Although the mechanism of metastasis has been elucidated in various ways and a number of new drugs for CRPC have been approved by the FDA, the development of metastasis and the formation of androgenindependent states remain tough issues to face in the clinic. Proteomic analysis has been used to detect metastasis-related proteins in PCa and showed that 58 proteins are differently expressed in lymph nodemetastatic PCa, compared with localized PCa. Six of these protein expression patterns, including the increased expression of e-FABP5, MCCC2, PPA2, Ezrin and SLP2 and the decreased expression of SM22, have a functional relation to $\mathrm{PCa}$ progression. ${ }^{27} \mathrm{HoxB} 3$ was found to promote $\mathrm{PCa}$ progression by transactivating CDCA3 ${ }^{28}$

CRPC is the result of the regrowth of PCa cells that have adapted to the hormone-deprived environment of the prostate. Many studies have focussed on CRPC progression and analysed the process from different angles; however, there is no simple answer to this question. Tissue PSAs may activate AR by enhancing the expression of ARA70, resulting in an androgen-refractory $\mathrm{PCa} .{ }^{29} \mathrm{In}$ accord with these findings, 5-aza-2'-deoxycytidine, which can effectively inhibit the methylation of the AR promoter, was reported to be an effective agent in the suppression of refractory progressions. ${ }^{30}$ Changes in miRNA are also involved in the progression of CRPC.

High-throughput Illumina sequencing was applied to find that some miRNAs are upregulated whereas others are downregulated in androgen-independent cell lines; this finding suggests that these miRNAs may be involved in the transition of $\mathrm{LNCaP}$ to an androgen-independent phenotype. $^{31}$ In investigating long, non-coding RNAs, we found a total of 137 long non-coding (nc) RNAs that were consistently upregulated or downregulated in PCa patients. We have confirmed two known lncRNAs and identified three novel long ncRNAs, namely, FR0257520, FR0348383 ${ }^{32}$ and PlncRNA-1, ${ }^{33}$ that are significantly associated with PCa. We postulate that long ncRNAs may have functions beyond transcriptional regulation, as PlncRNA-1 suppression resulted in a decrease of AR mRNA, proteins and AR downstream targets. This result suggests a reciprocal regulation of PlncRNA-1 and AR, revealing PlncRNA-1 as a potential therapeutic target for PCa.

\section{Therapeutic approaches}

Several therapeutic explorations aimed at three main aspects have been carried out: personalized therapy based on genetic factors, nanomedicine and traditional Chinese medicine. Future treatment approaches will utilize more specific and more personal treatment. Thalidomide has been tested in phase III trials for treating CRPC without satisfactory results. Some patients have a positive outcome to thalidomide treatment, but others do not respond at all. A possible explanation for the different responses to thalidomide, which we showed experimentally, is that sensitivity to thalidomide is positively correlated with CUL4A expression in a panel of prostate cell lines. CUL4A is a potential drug target of thalidomide, and high CUL4A expression is correlated with the effectiveness of thalidomide. ${ }^{34}$

In addition, several studies have focussed on biomarkers that can predict responses to docetaxel treatment in PCa. Chemosensitivity to 
docetaxel in three prostate cell lines has been demonstrated to be enhanced with the knockdown of p21-activated kinase 6. The knockdown of p21-activated kinase 6 expression caused an inhibitory effect on PCa growth and enhanced chemosensitivity to docetaxel. ${ }^{35}$ In addition, miR-21 was found to be upregulated in docetaxel-resistant cell lines, suggesting that miR-21 contributed to this resistance to docetaxel. ${ }^{36}$ In the future, these targets may serve as promising therapeutic approaches in sensitizing $\mathrm{PCa}$ to docetaxel treatment. Regarding the use of adjuvant therapy for PCa patients, a group of researchers identified two PCa susceptibility variants, rs16901979 and rs7931342, as prognostic predictors for survival after androgendeprivation therapy. These variants are associated with disease progression, PCa-specific mortality and all-cause mortality. This finding allows for the identification of high-risk patients who might benefit from appropriate adjuvant therapies after radical prostatectomy. ${ }^{37}$

Led by Xin Gao from the Third Affiliated Hospital of Sun Yat-sen University, investigators have tested nanoparticles intergraded with chemical active substances as promising imaging and therapeutic tools. Docetaxel-loaded oleic acid-coated hydroxyapatite nanoparticles can enhance the apoptosis of androgen-independent PCa cells by activating caspase-2. ${ }^{38}$ Another nanoparticle intergraded with docetaxel and superparamagnetic iron oxide showed both stable superparamagnetic behaviour and antiproliferative effects in PC3 cell lines. ${ }^{39}$ Moreover, previous studies have implemented a covalent layer-by-layer particle that is conjugated to a single-chain prostate stem cell antigen antibody and that can realize the targeted delivery to $\mathrm{PCa}$, offering a sustained release pattern in mice. ${ }^{40}$ Although study of these nanoparticles is still in the preliminary period, these nanoparticles have great potential for imaging and chemotherapy, considering the continuing improvements in nanotechnology.

Traditional Chinese medicine, the treasures left by our ancestors, is effective in combating cancer, but no specific mechanism has been identified. However, novel technologies may allow for the potential of components of traditional Chinese medicine as antitumour drugs to be elucidated. A group led by Mingyao Liu from East China Normal University has focussed on several components of traditional Chinese medicine as potential antitumour drugs that target different signalling pathways and inhibit tumour angiogenesis. Cucurbitacin E, ${ }^{41}$ plumbagin $^{42}$ and acetyl-11-keto-beta-boswellic acid $^{43}$ inhibit tumour angiogenesis by targeting the Vascular Endothelial Growth Factor Receptor 2 (VEGFR2)-related signalling pathway, while kisspeptin$10^{44}$ suppresses Sp1-mediated vascular endothelial growth factor expression and FAK/Rho GTPase activation. Since angiogenesis plays an important role in tumourigenesis, progression and metastasis, these components may act as a promising target therapy; still, more clinical trials should be carried out. Further, genistein, a major isoflavone constituent of soybeans, has been demonstrated to have anticancer effects. Researchers have found that genistein can effectively inhibit the transition of PCa cells from epithelial to mesenchymal, as well as their stemness properties. ${ }^{45}$

\section{FUTURE DIRECTION}

During the past few years, the Chinese public and government are beginning to pay more attention to $\mathrm{PCa}$ and have attached great importance to basic research related to $\mathrm{PCa}$, especially translational research. Governmental funding for PCa research has increased consistently, including the first National Basic Research Program of China (973 Program). Chinese scientists now face unprecedented opportunities and challenges. As mentioned above, novel gene fusions, copy number variances and long ncRNAs have been discovered in the
Chinese population. The vast discrepancy in prevalence of $\mathrm{PCa}$ between Chinese and Caucasian populations, as well as differences in each oncological pathway, urgently needs to be elaborated. Furthermore, it is hoped that in the near future, a Chinese PCa research cooperation network, including patient databases with concrete information and abundant patients, will be established. The results of GWAS should be extensively interpreted in the field of pharmacogenomics to identify better biomarkers for predicting drug efficacy and toxicity. Finally, next-generation sequencing may open a new window to a greater understanding of this disease, particularly in the Chinese population.

\section{AUTHOR CONTRIBUTIONS}

YHS provided original ideas and instructed the writing of this article. SCR and RC searched and summarized the literature. SCR and RC also wrote the manuscript.

\section{COMPETING FINANCIAL INTERESTS}

The authors declare no competing financial interests.

\section{ACKNOWLEDGMENTS}

This work was supported by grants from the National Basic Research Program of China (No. 2012CD518300), the National Natural Science Foundation of China (No. 81101946), and the Prostate Cancer Foundation Young Investigator Award, and Shanghai Pujiang Program (12PJD008).

1 Jemal A, Bray F, Center MM, Ferlay J, Ward E et al. Global cancer statistics. CA Cancer J Clin 2011; 61: 69-90.

2 Kamangar F, Dores GM, Anderson WF. Patterns of cancer incidence, mortality, and prevalence across five continents: defining priorities to reduce cancer disparities in different geographic regions of the world. J Clin Oncol 2006; 24: 2137-50.

3 Jian L, Xie LP, Lee AH, Binns CW. Protective effect of green tea against prostate cancer: a case-control study in southeast China. Int J Cancer 2004; 108: 130-5.

4 Shao Q, Ouyang J, Fan Y, Xie J, Zhou J et al. Prostate cancer in the senior men from rural areas in east district of China: contemporary management and 5-year outcomes at multi-institutional collaboration. Cancer Lett 2012; 315: 170-7.

5 Xu JF, Mo ZN, Ye DW, Wang ML, Liu F et al. Genome-wide association study in Chinese men identifies two new prostate cancer risk loci at 9q31.2 and 19q13.4. Nat Genet 2012; 44: 1231-5

6 Lu Y, Sun J, Kader AK, Kim ST, Kim JW et al. Association of prostate cancer risk with SNPs in regions containing androgen receptor binding sites captured by ChIP-On-chip analyses. Prostate 2012; 72: 376-85.

7 Lu Y, Zhang Z, Yu H, Zheng SL, Isaacs WB et al. Functional annotation of risk loci identified through genome-wide association studies for prostate cancer. Prostate 2011; 71: 955-63.

8 Aly $\mathrm{M}$, Wiklund $\mathrm{F}, \mathrm{Xu} \mathrm{J}$, Isaacs WB, Eklund $\mathrm{M}$ et al. Polygenic risk score improves prostate cancer risk prediction: results from the Stockholm-1 cohort study. Eur Urol 2011; 60: 21-8.

9 Garcia-Donas J, Esteban E, Leandro-Garcia LJ, Castellano DE, del Alba AG et al. Single nucleotide polymorphism associations with response and toxic effects in patients with advanced renal-cell carcinoma treated with first-line sunitinib: a multicentre, observational, prospective study. Lancet Oncol 2011; 12: 1143-50.

10 Prensner JR, Chinnaiyan AM. Oncogenic gene fusions in epithelial carcinomas. Curr Opin Genet Dev 2009; 19: 82-91.

11 Tomlins SA, Rhodes DR, Perner S, Dhanasekaran SM, Mehra R et al. Recurrent fusion of TMPRSS2 and ETS transcription factor genes in prostate cancer. Science 2005; 310: 644-8.

12 Mosquera JM, Mehra R, Regan MM, Perner S, Genega EM et al. Prevalence of TMPRSS2-ERG fusion prostate cancer among men undergoing prostate biopsy in the United States. Clin Cancer Res 2009; 15: 4706-11.

13 Ren S, Peng Z, Mao JH, Yu Y, Yin C et al. RNA-seq analysis of prostate cancer in the Chinese population identifies recurrent gene fusions, cancer-associated long noncoding RNAs and aberrant alternative splicings. Cell Res 2012; 22: 806-21.

14 Xue L, Mao X, Ren G, Stankiewicz E, Kudahetti SC et al. Chinese and Western prostate cancers show alternate pathogenetic pathways in association with ERG status. Am J Cancer Res 2012; 2: 736-44.

15 Magi-Galluzzi C, Tsusuki T, Elson P, Simmerman K, LaFargue C et al. TMPRSS2-ERG gene fusion prevalence and class are significantly different in prostate cancer of Caucasian, African-American and Japanese patients. Prostate 2011; 71: 489-97. 
16 Lee K, Chae JY, Kwak C, Ku JH, Moon KC. TMPRSS2-ERG gene fusion and clinicopathologic characteristics of Korean prostate cancer patients. Urology 2010 76: 1268. e7-13.

17 Miyagi Y, Sasaki T, Fujinami K, Sano J, Senga Y et al. ETS family-associated gene fusions in Japanese prostate cancer: analysis of 194 radical prostatectomy samples. Mod Pathol 2010; 23: 1492-8.

18 Mao X, Yu Y, Boyd LK, Ren G, Lin D et al. Distinct genomic alterations in prostate cancers in Chinese and Western populations suggest alternative pathways of prostate carcinogenesis. Cancer Res 2010; 70: 5207-12.

19 Mani RS, Chinnaiyan AM. Triggers for genomic rearrangements: insights into genomic, cellular and environmental influences. Nat Rev Genet 2010; 11: 819-29.

20 Prensner JR, Rubin MA, Wei JT, Chinnaiyan AM. Beyond PSA: the next generation of prostate cancer biomarkers. Sci Trans/ Med 2012; 4: 127rv3.

21 Zheng J, Liu F, Lin X, Wang X, Ding Q et al. Predictive performance of prostate cancer risk in Chinese men using 33 reported prostate cancer risk-associated SNPs. Prostate 2012; 72: 577-83.

22 Cao DL, Ye DW, Zhang HL, Zhu Y, Wang YX et al. A multiplex model of combining genebased, protein-based, and metabolite-based with positive and negative markers in urine for the early diagnosis of prostate cancer. Prostate $2011 ; 71$ : 700-10.

23 Chen ZH, Zhang GL, Li HR, Luo JD, Li ZX et al. A panel of five circulating microRNAs as potential biomarkers for prostate cancer. Prostate 2012; 72: 1443-52.

24 Ren S, Ouyang DY, Saltis M, Xu LH, Zha QB et al. Anti-proliferative effect of 23,24 dihydrocucurbitacin $\mathrm{F}$ on human prostate cancer cells through induction of actin aggregation and cofilin-actin rod formation. Cancer Chemother Pharmacol 2012, 70: 415-24.

25 Guo YF, Li FH, Xie SW, Xia JG, Fang $\mathrm{H}$ et al. Value of contrast-enhanced sonographic micro flow imaging for prostate cancer detection with t-PSA level of 4-10 ng/mL. Eur J Radiol 2012; 81: 3067-71.

26 Bai Y, Wang MY, Han YH, Dou SW, Lin Q et al. Susceptibility weighted imaging: a new tool in the diagnosis of prostate cancer and detection of prostatic calcification. PLOS One 2013; 8: e53237

27 Pang J, Liu WP, Liu XP, Li LY, Fang YQ et al. Profiling protein markers associated with lymph node metastasis in prostate cancer by DIGE-based proteomics analysis. J Proteome Res 2010; 9: 216-26.

28 Chen J, Zhu S, Jiang N, Shang Z, Quan C et al. HoxB3 promotes prostate cancer cell progression by transactivating CDCA3. Cancer Lett 2013; 330: 217-24.

29 Niu Y, Yeh S, Miyamoto H, Li G, Altuwaijri S et al. Tissue prostate-specific antigen facilitates refractory prostate tumor progression via enhancing ARA70-regulated androgen receptor transactivation. Cancer Res 2008; 68: 7110-9.

30 Tian J, Lee SO, Liang L, Luo J, Huang CK et al. Targeting the unique methylation pattern of androgen receptor (AR) promoter in prostate stem/progenitor cells with 5-aza-2'-deoxycytidine (5-AZA) leads to suppressed prostate tumorigenesis. J Biol Chem 2012; 287: 39954-66.

31 Xu G, Wu J, Zhou L, Chen B, Sun Z et al. Characterization of the small RNA transcriptomes of androgen dependent and independent prostate cancer cell line by deep sequencing. PLoS One 2010; 5: e15519.
32 Ren SC, Peng ZY, Mao JH, Yu YW, Yin CJ et al. RNA-seq analysis of prostate cancer in the Chinese population identifies recurrent gene fusions, cancer-associated long noncoding RNAs and aberrant alternative splicings. Cell Research 2012; 22 806-21.

33 Cui Z, Ren S, Lu J, Wang F, Xu W et al. The prostate cancer-up-regulated long noncoding RNA PIncRNA-1 modulates apoptosis and proliferation through reciprocal regulation of androgen receptor. Urol Oncol; e-pub ahead of print 19 January 2012

34 Ren S, Xu C, Cui Z, Yu Y, Xu W et al. Oncogenic CUL4A determines the response to thalidomide treatment in prostate cancer. J Mol Med (Berl) 2012; 90: 1121-32.

35 Wen X, Li X, Liao B, Liu Y, Wu J et al. Knockdown of p21-activated kinase 6 inhibits prostate cancer growth and enhances chemosensitivity to docetaxel. Urology 2009; 73: 1407-11.

36 Shi GH, Ye DW, Yao XD, Zhang SL, Dai B et al. Involvement of microRNA-21 in mediating chemo-resistance to docetaxel in androgen-independent prostate cancer PC3 cells. Acta Pharmacol Sin 2010; 31: 867-73.

37 Bao BY, Pao JB, Huang CN, Pu YS, Chang TY et al. Significant associations of prostate cancer susceptibility variants with survival in patients treated with androgendeprivation therapy. Int J Cancer 2012; 130: 876-84.

38 Luo Y, Ling Y, Guo W, Pang J, Liu W et al. Docetaxel loaded oleic acid-coated hydroxyapatite nanoparticles enhance the docetaxel-induced apoptosis through activation of caspase- 2 in androgen independent prostate cancer cells. $J$ Contro Release 2010; 147: 278-88

39 Ling Y, Wei K, Luo Y, Gao X, Zhong S. Dual docetaxel/superparamagnetic iron oxide loaded nanoparticles for both targeting magnetic resonance imaging and cancer therapy. Biomaterials 2011; 32: 7139-50.

40 Gao X, Luo Y, Wang Y, Pang J, Liao C et al. Prostate stem cell antigen-targeted nanoparticles with dual functional properties: in vivo imaging and cance chemotherapy. Int J Nanomedicine 2012; 7: 4037-51.

41 Dong $Y$, Lu B, Zhang X, Zhang J, Lai L et al. Cucurbitacin E, a tetracyclic triterpenes compound from Chinese medicine, inhibits tumor angiogenesis through VEGFR2-mediated Jak2-STAT3 signaling pathway. Carcinogenesis 2010 31: 2097-104.

42 Lai L, Liu J, Zhai D, Lin Q, He L et al. Plumbagin inhibits tumour angiogenesis and tumour growth through the Ras signalling pathway following activation of the VEGF receptor-2. Br J Pharmacol 2012; 165: 1084-96.

43 Pang X, Yi Z, Zhang X, Sung B, Qu W et al. Acetyl-11-keto-beta-boswellic acid inhibits prostate tumor growth by suppressing vascular endothelial growth factor receptor 2-mediated angiogenesis. Cancer Res 2009; 69: 5893-900.

44 Cho SG, Yi Z, Pang X, Yi T, Wang Y et al. Kisspeptin-10, a KISS1-derived decapeptide, inhibits tumor angiogenesis by suppressing Sp1-mediated VEGF expression and FAK Rho GTPase activation. Cancer Res 2009; 69: 7062-70.

45 Zhang L, Li L, Jiao M, Wu D, Wu K et al. Genistein inhibits the stemness properties of prostate cancer cells through targeting Hedgehog-Gli1 pathway. Cancer Lett 2012 323: 48-57. 